\title{
Research on Capacity Configuration and Optimal Operation of Microgrid Energy Storage under Demand Management
}

\author{
Wensheng $\mathrm{Li}^{1}$, Donglei Sun ${ }^{1}$, Yajin $\mathrm{Li}^{2}$, Xian Wang ${ }^{1}$, Rui Liu ${ }^{l}$, Dayang Yu ${ }^{2}$ \\ ${ }^{1}$ Economic Research Institute of State Grid Shandong Electric Power Co., Ltd., Jinan 250022, China \\ ${ }^{2}$ School of Electrical Engineering, Shandong University, Jinan 250061, Shandong, China
}

\begin{abstract}
In order to reduce the energy consumption cost, considering the influence of the correlation of photovoltaic output, load demand and peak valley TOU price in different periods on the optical storage capacity configuration, the system operation strategy and capacity configuration principle are determined based on the peak valley TOU price and energy storage charge state information and the actual size of photovoltaic output and load.Integrated with the historical load, time of use electricity price, state of charge, charge discharge power and other constraints of the energy storage system, the minimum objective function is utilized to optimize the output power of energy storage. The effectiveness of the model is verified by an example. The sensitivity analysis shows that different demand tariff rules affect the user demand declaration strategy. The energy storage system planning selects the light storage combination with appropriate capacity according to the demand tariff rules and the change of energy storage investment cost, which has practical engineering value.
\end{abstract}

Keywords: Capacity configuration; energy consumption cost; demand electricity price; optimal scheduling.

\section{Introduction}

The dispatching control of energy storage and peak electricity price on the user side of microgrid can effectively reduce the user peak load and reduce the capacity electricity charge. For the power grid, it can bring the reduction of peak shaving cost caused by the reduction of load fluctuation and the reduction of system capacity caused by the reduction of maximum load. However, limited by the cost of energy storage capacity, under the price policy and market environment, how to balance the power consumption cost and energy storage investment of users, minimize the power consumption cost of microgrid users, and realize the energy storage evaluation of industrial large users with demand management and peak valley arbitrage need to be further studied.

Scholars have studied the optimal allocation and investment economy of energy storage applications. Reference [9] studies the cost and return on investment model of user side battery energy storage in the whole life cycle. Reference [10] establishes the investment, operation and maintenance cost of energy storage power station and the income model under different applications, and considers the charge and discharge depth and service life of battery. Document [11] proposed the operation mode of the battery energy storage system on the user side of the industrial park, considered the charge discharge operation under different energy storage strategies, and established the income and cost model of energy storage operation, but only carried out peak valley price difference arbitrage without considering the maximum demand control. Reference [12] establishes a month before and day before two-stage model for energy storage optimization, which takes the minimum total electricity charge and the maximum peak valley arbitrage as the objective function to calculate the charge and discharge power of output energy storage, but does not consider the situation that the number of cycles will affect the battery loss.

To sum up, the main source of income of energy storage investment on the user side is the saved user electricity bill, but most models do not fully consider the impact of parameters, performance characteristics and life cycle of energy storage system on its cost and income value. In order to ensure the best economic benefit in the operation of energy storage, an energy storage evaluation model is established with the minimum comprehensive cost of users, to evaluate the economy of users installing energy storage and optimize the energy storage configuration; The annual and intra day optimization models of energy storage are constructed. In the annual optimization, determine the monthly maximum demand reported by the user; In the intra day optimization, the daily energy storage charge and discharge power is optimized based on the maximum demand constraint. At the same time, this paper further analyzes the return on investment and user 
power cost of energy storage under different sensitive parameters, and verifies the economy of the model.

\section{Demand management}

Under the two-part electricity price, the electricity charge is mainly composed of basic electricity charge and electricity charge. The demand electricity charge belongs to one of the ways of basic electricity charge. The demand management is to reasonably control the user load so that the maximum power does not exceed the maximum power of contract electricity. Therefore, by making full use of the flexibility of energy storage power regulation, discharging at the peak of power consumption and controlling the maximum demand value of load, so as to reduce the basic electricity charge is an effective means to improve the income.

The basic electricity charge to be paid by the user is the maximum demand reported in the current month multiplied by the unit price (different in different regions). On this basis, when the actual maximum value exceeds 1.05 times the approved value, the part exceeding the approved value will be doubled. The electricity charge model per unit demand is:

$$
\tilde{c}=c(x, \tilde{D})=\left\{\begin{array}{c}
p_{1} x+p_{2}(\tilde{D}-k x), k x<\tilde{D} \leq C \\
p_{1} x, \tilde{D} \leq k x
\end{array}\right.
$$

Where, $\mathrm{C}$ is the transformer capacity; $D$ is the actual maximum demand; $x$ is the maximum demand, which shall not be lower than a certain distribution transformer capacity; $\mathrm{k}$ is the threshold of punitive price, $\mathrm{k}=1.05$ according to the current regulations; $\mathrm{p} 1$ and $\mathrm{p} 2$ are the demand price of the actual maximum demand within 1.05 times the reported maximum demand and the demand price of the part exceeding 1.05 times the reported maximum demand respectively. According to the current regulations, $\mathrm{p} 2 / \mathrm{p} 1=2$.

\section{Energy storage capacity configuration}

\subsection{Minimum objective function of user power cost}

The objective function of energy storage capacity allocation is to minimize the user's power consumption cost in the research period $\mathrm{T}$, that is, the sum of electricity charge and demand charge minus the minimum demand response income, which can be expressed as:

$$
\begin{aligned}
& \min C=\sum_{t=1}^{T}\left(C_{1}+C_{2}+C_{3}\right) \cdot \Delta t+C_{4} \\
& C_{1}=\left(P_{l t}-P_{v t}+P_{c h, t}-P_{d i s, t}\right) \cdot p_{t} \\
& C_{2}=P_{v t} \frac{C_{p v}}{P_{p v} \cdot H \cdot N_{p v}} \\
& C_{3}=P_{d i s, t} \frac{C_{B E S S}}{E_{B E S S} \cdot n_{b e s s}} \\
& C_{4}=\alpha \cdot P_{\text {demand }} \cdot T_{n}
\end{aligned}
$$

Where, $\mathrm{C}$ is the total power consumption cost of microgrid users; $C_{1}$ represents the power purchase cost of microgrid users from the power grid; $C_{2}$ represents the cost of photovoltaic power generation; $C_{3}$ represents the energy storage and discharge cost. $\mathrm{T}$ is the number of periods in a research cycle and the duration interval of each period; $C_{4}$ indicates the electricity demand of the user; $\mathrm{R}$ represents the benefit of user participation in demand response.

Where, $P_{l t}$ represents the power grid load in period $\mathrm{t} ; P_{v t}$ is the photovoltaic power generation power in period $\mathrm{T}$, $P_{c h, t} 、 P_{d i s, t}$ which respectively represents the charging power and discharge power of energy storage battery in period $\mathrm{t}$, and $p_{t}$ is the time-of-use price of power grid.

Where, $C_{p v}$ represents the overall photovoltaic investment cost (yuan), $P_{p v}$ represents the photovoltaic installed capacity $(\mathrm{kw}), \mathrm{H}$ represents the maximum utilization hours of photovoltaic year, and $N_{p v}$ represents the photovoltaic operation life.

Where, $C_{B E S S}$ represents the overall investment cost of energy storage (yuan), $E_{B E S S}$ represents the rated capacity of energy storage configuration (KWh) and $n_{\text {bess }}$ represents the number of energy storage cycles.

Where, $P_{\text {demand }}$ represents the maximum demand of users and $\alpha$ is the electricity charge per unit demand.

\subsection{Constraint condition}

Capacity of the previous time + charging and discharging power of the current period $*$ time $=$ capacity of the current time.

$$
E_{t}=E_{t-1}+\eta_{c h} \cdot P_{c h, t} \cdot \Delta t-\Delta t \cdot P_{d i s, t} / \eta_{d}
$$

Where, $E_{t}$ and $E_{t-1}$ represent the remaining capacity of the battery in t period and $\mathrm{t}-1$ period, and $\eta_{c h} \eta_{d}$ are the charging efficiency and discharge efficiency of the energy storage battery respectively.

2) The inequality constraint of the remaining battery power in period $t$ is:

$$
E_{\min } \leq E_{t} \leq E_{B E S S}
$$

Where, $E_{B E S S}$ represents the rated capacity of energy storage configuration and $E_{\min }$ is the lower limit of energy storage capacity.

3) The charging and discharging power of energy storage shall not exceed the limit.

$$
\begin{aligned}
& 0 \leq P_{c h, t} \leq P_{\max } \\
& 0 \leq P_{d i s, t} \leq P_{\max }
\end{aligned}
$$

Where, $P_{\max }$ is the maximum charge discharge power limit of the energy storage system.

Regardless of the grid connection of energy storage, the configuration of energy storage is completely used to 
absorb photovoltaic and reduce user load. Therefore, the maximum charge and discharge power of the energy storage system only needs to meet the power imbalance within a certain period $\mathrm{T}$ within the assessment cycle $\mathrm{t}$, that is:

$$
P_{\max } \leq\left|\max _{t \in[1, T]}\left[P_{l t}-P_{v t}\right]\right|
$$

4) Maximum demand constraint

Guided by peak valley electricity price and demand response compensation, energy storage may form new load spikes, or even exceed the original maximum demand, resulting in the increase of demand electricity charge. Therefore, this paper introduces the maximum demand constraint.

$$
\max \left(P_{l t}-P_{v t}+P_{c h, t}-P_{d i s, t}\right) \leq 1.05 P_{\text {demand }}
$$

The above model is a dynamic programming problem. Equations (3) to (7) constitute the constraints of the objective function, including the constraints of battery power, charging demand, charging power, etc. In the model, $P_{c h, t} 、 P_{\text {dis }, t}, E_{\text {BESS }}, P_{\text {demand }}$ are decision variables, and other parameters are known quantities.

The above model is a dynamic programming problem. This paper establishes the model in Python and calls the optimization function library to solve it.

\subsection{Evaluating indicator}

This paper uses the return on investment index of energy storage in the whole life cycle to evaluate whether users are suitable for installing energy storage.

$$
R=B / C_{B E S S}
$$

Where, $\mathrm{R}$ represents the return on investment, $\mathrm{B}$ represents the return on energy storage, that is, the electricity saved before and after energy storage, and $C_{B E S S}$ represents the overall investment cost of energy storage (yuan).

\section{Analysis of case}

\subsection{Basic data}

Taking the power consumption data of an industrial user as an example, the typical daily power load is shown in Figure 1.

According to the available photovoltaic sites, the maximum installed capacity of photovoltaic is 4.5342mwp. According to the calculation, the typical output curve of photovoltaic is shown in Figure 2. The annual photovoltaic power generation is $5562 \mathrm{mwh}$ and the annual utilization hours of photovoltaic are $1235.9 \mathrm{~h}$. The photovoltaic investment cost is 3500 yuan / $\mathrm{kW}$, and the operation and maintenance cost is not considered.

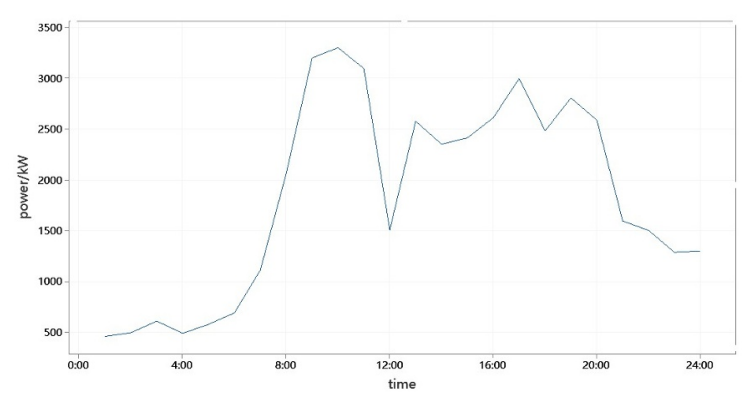

Fig. 1. Typical daily load curve

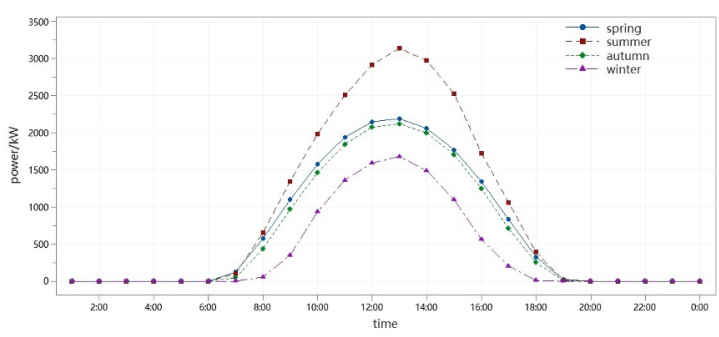

Fig. 2. Typical output curve of four seasons photovoltaic

The peak valley time division of Shandong Province is shown in Table 1 . The time of use electricity price is: peak electricity price of 1.0102 yuan / $\mathrm{kWh}$, peak electricity price of 0.8948 yuan / $\mathrm{kWh}$, normal electricity price of 0.6062 yuan / $\mathrm{kWh}$ and valley electricity price of 0.3177 yuan $/ \mathrm{kWh}$. The maximum demand electricity price of Shandong power grid is 38 yuan $/ \mathrm{kW} \cdot$ month.

The type of energy storage battery is lithium iron phosphate, and the battery price is 900 yuan / $\mathrm{kWh}$. The battery cycle life is 1500 times, and the battery charge / discharge efficiency is $92 \%$.

\subsection{Simulation analysis}

Combined with the actual load power data of the user, this paper calculates the optimal energy storage capacity configuration of the user, considers the impact of lithium iron phosphate battery charge and discharge on capacity loss, and uses the objective function in Section 2.1 to evaluate the economy of energy storage after participating in demand management.

The scheduling interval is 1 hour. Assuming that the output power of photovoltaic and load is constant within 1 hour, the fluctuation of four typical days in spring, summer, autumn and winter represents the change of one year, and 8760 hours of the whole year are analyzed in combination with the days of different typical days.

When energy storage participates in peak valley arbitrage under TOU price, the optimal operation scheme is solved. Take the load data from June 29 to July 28 for the test, and set the equivalent charge and discharge times $\mathrm{M}=2$. Figure 3 shows the load curve comparison before and after typical daily energy storage participating in peak valley arbitrage. The abscissa is the sampling point with a sampling interval of $15 \mathrm{~min}$. Solve the model to obtain the energy storage operation strategy with the minimum electricity charge. The optimal configuration of energy 
storage is $2347.5 \mathrm{kwh}$ and the reported maximum demand is $2235.5 \mathrm{kw}$.

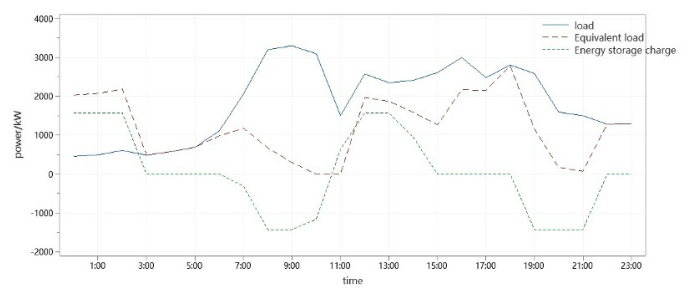

Fig. 3. Typical daily energy storage in spring and equivalent load curve after peak valley TOU price optimization

It can be seen from the figure that under the optimized energy storage charge and discharge strategy, the energy storage is attracted by the price difference between peak and valley. It is selected to charge and discharge in valley and peace periods and peak periods. By reducing the peak load, the final power consumption curve is smoothed as much as possible while earning the income from peak cutting and valley filling.

It can be seen from the analysis in Figure 4 that the rated power of energy storage and the monthly comprehensive cost of users tend to decrease first and then increase, while the expected income is on the contrary. This is because after the energy storage is increased to a certain capacity, the growth rate of monthly electricity saving is not enough to offset the growth rate of energy storage cost, resulting in the increase of comprehensive cost and the decrease of expected income. It can be seen intuitively from the figure that when the energy storage power is $998 \mathrm{kw}$, the comprehensive cost of users is the lowest and the expected income is the largest.

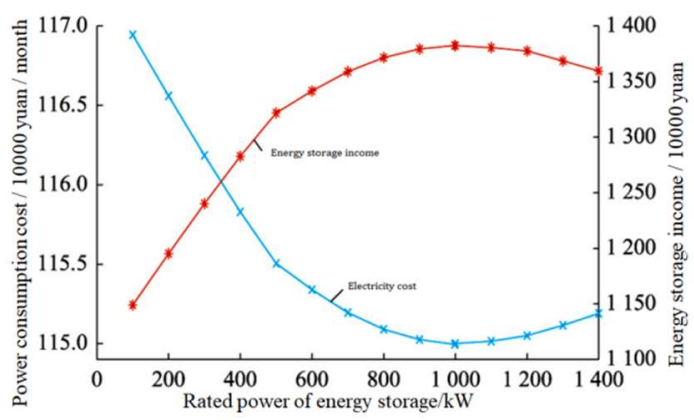

Fig. 4. Impact of energy storage allocation on users' economic benefits

\subsection{Sensitivity analysis}

Under different demand electricity prices, analyze the declaration of maximum demand. As shown in Figure 5, the four demand electricity tariff curves are the electricity tariff curves when $\mathrm{P} 1=[42,39,33,30]$ from top to bottom. The optimal solution of the maximum contract demand is marked at the bottom of each curve. It can be seen from the figure that the change of unit demand electricity price can not affect the user's contract maximum demand declaration strategy, but can only affect the user's demand electricity fee expenditure, and this impact is in direct proportion to the unit demand electricity price.

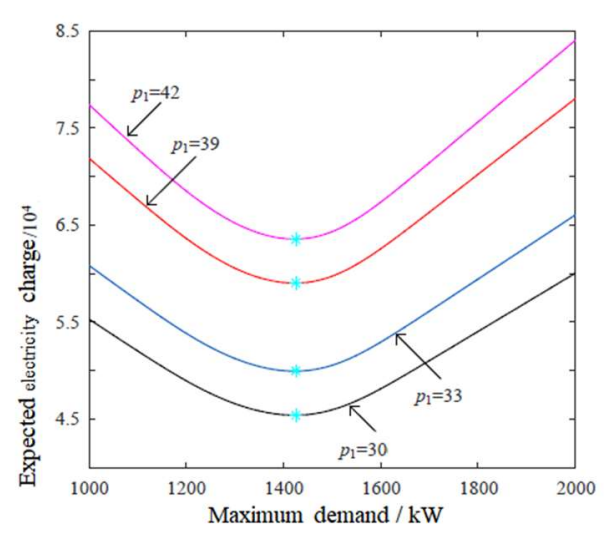

Fig. 5. Demand tariff curve under different demand tariff

In the current regulations, the part where the actual maximum demand exceeds 1.05 times of the contract maximum demand will be doubled, that is, the threshold of punitive charge is $\mathrm{k}=1.05$. In this section, users in the same case are simulated under the same other conditions and only the punitive charging threshold $\mathrm{K}$ changes, so as to intuitively show the impact of the change of punitive charging threshold on users' demand declaration. The simulation results are shown in Figure 6.

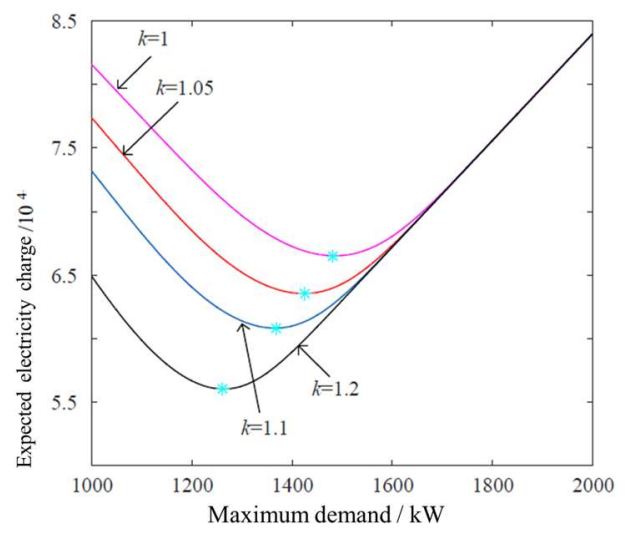

Fig. 6. Demand electricity charge curve under different penalty rates

The four demand tariff curves are the tariff curves of $\mathrm{k}=$ $[1,1.05,1.1,1.2]$ from top to bottom. The optimal solution of the maximum contract demand is marked at the bottom of each curve. It can be seen from the figure that the adjustment of punitive charge threshold $\mathrm{K}$ can affect the declared value of users and the minimum value of electricity demand. The greater the punitive charge rate, the smaller the declared value of the user's maximum contract demand, and the smaller the corresponding minimum demand electricity charge.

\section{Conclusion}

Taking the minimum comprehensive cost of users as the objective function, this paper establishes an energy storage evaluation model. The example shows that it is suitable to install energy storage for large industrial users 
with load peak and large peak valley difference in the daytime; For users with relatively flat load curve, it is not suitable to install energy storage.

A two-stage energy storage optimization model is established in this paper. In the pre month optimization, the minimum total electricity charge of users is taken as the objective function to determine the reported maximum demand. In the intra day optimization, the maximum peak valley arbitrage of users is taken as the objective function to optimize the energy storage charge and discharge power and further improve the economic benefits of users. At the same time, through sensitivity analysis, this paper discusses the impact of the adjustment of demand tariff rules on users' declaration strategy and demand tariff from the perspective of power companies, and discusses the changes of demand tariff rules parameters including unit demand tariff and punitive charge threshold, which provides valuable conclusions and suggestions for the formulation and adjustment of demand tariff rules of power grid companies.

\section{Acknowledgment}

This work was supported by the science and technology projects of Economic Research Institute of State Grid Shandong Electric Power Co., Ltd. (SGSDJY00JPJS2000227).

\section{References}

1. CHEN Changqing, CHEN Changqing.Research on grid energy storage and photovoltaic coordination planning with flexible load[J].Power System Protection and Control, 2021,49(4):169-177

2. LI Jianlin, MA Huimeng, HUI Dong. Present development condition and trends of energy storage technology in the integration of distributed renewable energy $[\mathrm{J}]$. Transactions of China Electrotechnical Society, 2016, 31(14) :1-10.

3. ZHANG Mingxia, YAN Tao, LAI Xiaokang, et al. Technology vision and route of energy storage under new power grid function configuration[J]. Power system technology, 2018, 42(5) :1 370-1 377.

4. BARTON J, INFIELD D. Energy storage and its use with intermittent renewable energy[J]. IEEE Transactions on Energy Conversion, 2004, 19(2) :441-448.

5. FAN Haifeng, YU Zhipeng, LIU Wenlong, et al. A demand assessment approach of energy storage for participating in fast frequency regulation of power system $[\mathrm{J}]$. Electric Power Engineering Technology, 2019, 38(2) :18-24.

6. XU Haihua, WANG Xudong, ZHU Xingyang, et al. Research on the optimal configuration of energy storage in user side integrated energy system[J]. Power Demand Side Management, 2020, 22(2) :1320.

7. LI Hua, WANG Simin, GAO Jie. Research on energy storage control strategy of wind solar storage combined generation system based on tracking plan
[J]. Electrical \& Energy Management Technology, 2019(4) : 71-78.

8. GUO Li, XUE Guiyuan, WU Chen, et al. Research on economic evaluation of energy storage applied to load shift [J]. Power Demand Side Management, 2019, 21(5):31-34.

9. XUE Jinhua, YE Jilei, TAO Qiong, et al. Economic feasibility of user-side battery energy storage based on whole-life cycle cost model[ J]. Power System Technology, 2016, 40 (8) : 2 471-2 476.

10. XIONG Xiong, YANG Rengang, YE Lin, et al. Economic evaluation of large scale energy storage allocation in power demand side[J]. Transactions of China Electrotechnical Society, 2013, 28(9) : 224230.

11. JIA Xuecui, LI Xiangjun, WANG Huanling, et al. Re - search on consistency assessment method for energy storage battery based on operating data fusion[J]. Distribution \& Utilization, 2017, 34(4) : 29-35.

12. CHEN Lijuan, WU Tiantian, LIU Huibo, et al. Demand management based two- stage optimal storage model for large users[J]. Automation of Electric Power System, 2019, 43(1) :194-200. 